\title{
Expression of ADAMTS4 and ADAMTS5 in longissimus dorsi muscle related to meat tenderness in Nanyang cattle
}

\author{
X.H. Zhang ${ }^{1}$, Y.X. Qi ${ }^{1}$, X. Gao ${ }^{2}$, J.Y. $\mathrm{Li}^{2}$ and S.Z. Xu' \\ ${ }^{1}$ College of Animal Science, Henan University of Science and Technology, \\ Luoyang, He'nan, China \\ ${ }^{2}$ Institute of Animal Science, Chinese Academy of Agricultural Sciences, \\ Beijing, China \\ Corresponding authors: X.H. Zhang / S.Z. Xu \\ E-mail: zhangxiaohui78@126.com / simmenta@vip.sina.com
}

Genet. Mol. Res. 12 (4): 4639-4647 (2013)

Received August 1, 2012

Accepted February 4, 2013

Published October 18, 2013

DOI http://dx.doi.org/10.4238/2013.October. 18.2

\begin{abstract}
The ADAMTS4 and ADAMTS5 are secreted proteases, which can cleave aggrecan, brevican and versican to regulate rebuilding of the extracellular matrix. We analyzed the ADAMTS4 and ADAMTS5 gene expression patterns in longissimus dorsi muscle at intervals from 135 days fetal age to 30 months old by qRT-PCR in Nanyang cattle. Expression of ADAMTS4 was significantly higher in 135 and 185-day-old fetuses than at other stages, while expression of ADAMTS5 decreased during development. The promoter regions of ADAMTS4 and ADAMTS5 were cloned and the transcription factor binding sites were analyzed with bioinformatic methods. Twelve and six potential transcription factor binding sites were found in the promoter regions of ADAMTS4 and ADAMTS5 genes, respectively. Three transcription factors (MZF1, $\mathrm{C} / \mathrm{EBPb}$, and NF-kap) were selected to analyze the expression pattern during the development of the longissimus dorsi muscle. MZF1 was significantly co-expressed with ADAMTS4, while C/EBPb expression was significantly negatively associated with that of ADAMTS4. We concluded that the expression of ADAMTS4 is positively regulated by MZF1 and negatively regulated by $\mathrm{C} / \mathrm{EBPb}$. We examined the relationships of ADAMTS4 and ADAMTS5 expression with tenderness of longissimus
\end{abstract}


dorsi muscle; ADAMTS4 was significantly and negatively correlated with meat tenderness. We conclude that ADAMTS4 participates in the regulation of muscle development in cattle.

Key words: Nanyang cattle; ADAMTS4; ADAMTS5; Tenderness; Muscle development

\section{INTRODUCTION}

Meat tenderness is very important to meat quality and consumer acceptance. Tenderness is based on ease of chewing, which is attributed to many factors. Among them, the fibrous nature of muscle contributes to chewing resistance. Among many components of muscle fiber, protein is the most important one. Muscle proteins are categorized as sarcoplasmic, myofibrillar and stromal proteins on the basis of their solubility. Stromal proteins include proteins of the extracellular matrix (ECM), which are very fibrous and insoluble. The ECM contributes to the strength of muscle but also makes it tough. The tenderness of meat is obviously influenced by collagens of the muscle, which are the main component of ECM (Gillies and Lieber, 2011). The proteoglycans are another main component in ECM, which trap water and affect the viscoelastic properties of the tissue, helping the tissue resist compressive forces (Yanagishita, 1993). The expression of proteoglycans in muscle is related to myocyte growth and fatness trait in pigs (Cánovas et al., 2010). Aggrecan is the major proteoglycan in ECM and is responsible for the ability to resist compression by hydrating and swelling against the type II collagen scaffold (Roughley, 2001). The expression of aggrecan is high in muscle tissue, and this indicates that aggrecan may affect the tenderness of meat.

The ECM undergoes dynamic rebuilding and the ADAMTS superfamily plays important roles in this procession. The ADAMTS superfamily includes 19 metalloproteases, which have important roles in the maturation of precursor proteins as well as turnover of ECM (Apte, 2004; Porter et al., 2005). ADAMTS proteinases are a group of secreted enzymes, which hydrolyze the proteins of ECM and are involved in ectodomain shedding or activation of diverse cell surface molecules, including growth factors and adhesion receptors to regulate cell proliferation, differentiation, migration and apoptosis in muscle tissue (Seals and Courtneidge, 2003). The most studied aggrecanases are ADAMTS4 and ADAMTS5, which can also cleave the chondroitin sulfate proteoglycan brevican, predominantly expressed in the central nervous system (Matthews et al., 2000), and versican, present in blood vessels (Sandy et al., 2001). The expression of ADAMTS4 and ADAMTS5 is related to arthritis (Fosang and Rogerson, 2010; Durigova et al., 2012) and cell migration in cancer (Hojilla et al., 2008). The effects of ADAMTS4 and ADAMTS5 on ECM rebuilding in cartilage tissue and vascular tissue have been studied in depth, but such study is rarely reported in muscle tissue. In this paper, the expression of ADAMTS4 and ADAMTS5 was analyzed during the development of the longissimus dorsi muscle and the relationship of their expression levels with meat tenderness was analyzed in Nanyang cattle. The results of this study reveal the new function of ADAMTS4 and ADAMTS5 in controlling meat quality in cattle.

\section{MATERIAL AND METHODS}

\section{Tissue collection, RNA isolation and cDNA synthesis}

All experimental and surgical procedures were approved by the Biological Studies 
Animal Care and Use Committee, Henan Province, Peoples Republic of China. Nine pregnant cows were submitted to caesarean section to collect the fetuses at 135, 185 and 280 days of gestation (three pregnant cows from each stage), and the longissimus dorsi muscles from each fetus were dissected and snap-frozen in liquid nitrogen for gene expression analysis. Postnatal longissimus dorsi muscle tissue samples (four animals at each age of 3, 6, 12, 18, 24,30 months old) were collected in the experimental abattoir. A total of 24 Nanyang bulls aged 20 months were selected to analyze the correlation of meat quality with gene expression. The longissimus dorsi muscle was excised from each animal within 30 min post-mortem in an experimental abattoir. After dissection, samples were quickly frozen in liquid nitrogen and stored at $-80^{\circ} \mathrm{C}$ for later analysis.

Total RNA was isolated from all the samples using the RNAiso Plus kit (Takara, Dalian, China). RNA quality was determined by $1.2 \%$ agarose gel electrophoresis and absorbance at $260 \mathrm{~nm}$ in a Nanodrop ND-1000 ${ }^{\circledR}$ Spectrophotometer (NanoDrop, USA). After DNase I (Takara) treatment, total RNA was reverse transcribed to cDNA using the PrimeScript ${ }^{\mathrm{TM}}$ RT reagent kit (Takara) following the manufacturer instructions.

\section{Quantitative (q) RT-PCR analysis}

qRT-PCR was performed on an $\mathrm{iQ}^{\mathrm{TM}}$ Real Time PCR Detection System (Bio-Rad) using SYBR ${ }^{\circledR}$ Premix Ex Taq ${ }^{\mathrm{TM}}$ II (Takara). Thermal cycling consisted of an initial step at $95^{\circ} \mathrm{C}$ for $4 \mathrm{~min}$ followed by 42 cycles at $95^{\circ} \mathrm{C}$ for $30 \mathrm{~s}$, annealing for $30 \mathrm{~s}$ and extension/fluorescence acquisition at $72^{\circ} \mathrm{C}$ for $30 \mathrm{~s}$. Glyceraldehyde-3-phosphate dehydrogenase gene (GAPDH) was chosen as the reference gene for normalization of all data because it is expressed more stably in muscle tissues. Each qRT-PCR reaction (in $20 \mu \mathrm{L}$ ) contained $10 \mu \mathrm{L}$ SYBR $^{\circledR}$ Premix Ex $\mathrm{Taq}^{\mathrm{TM}} \mathrm{II}, 0.7 \mu \mathrm{L}$ each primer (Table1), $1 \mu \mathrm{L}$ normalized template cDNA, and $7.6 \mu \mathrm{L}$ ultrapure water. The qRT-PCR measurements were performed in triplicate for each cDNA sample, and gene expression was quantified relative to GAPDH expression using the $2^{-\Delta \Delta \mathrm{Ct}}$ method (Livak and Schmittgen, 2001). Gene expression ratios were normalized to the GAPDH gene in the same sample.

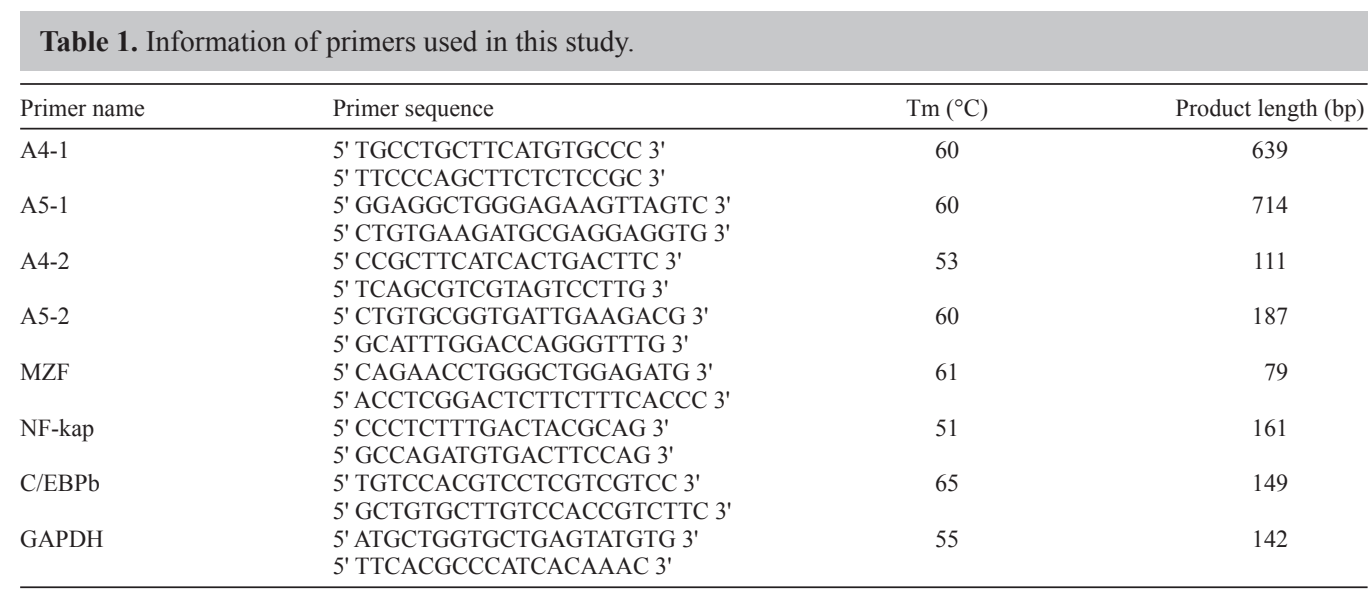

All the primers were designed with the Premier 5.0 software and synthesized in Sangon Biotech (Shanghai, China) Co. Ltd. 


\section{Cloning and transcription factor binding site analysis of 5'UTR of ADAMTS4 and ADAMTS5 genes}

The 5'UTR of the ADAMTS4 and ADAMTS5 genes were cloned by A4-1 and A5-1 primers (Table1) from cattle genomic DNA. The PCR products were separated by gel electrophoresis and extracted from a gel slice (Takara), and then cloned into pMD-19T vector and sequenced.

Regions spanning 500 bp upstream and 100 bp downstream of the transcription start site were regarded as the promoter region (Yang and Elnitski, 2008). The potential transcription factor (TF) binding sites were analyzed by the online TFSEARCH tool (http:// www.cbrc.jp/research/db/TFSEARCH.html). The options for TFSEARCH were set as matrix, vertebrate and the threshold value, and more than 90 because of using only highly conserved regions.

\section{Meat tenderness measurement}

Meat tenderness measurements were conducted using a muscle tenderness device (CLM3, China) with a Warner-Bratzler attachment in accordance with the method described by Iwanowska (Iwanowska et al., 2011). Prior to value measurements of shear force, samples were cut into slices $25-30 \mathrm{~mm}$ thick, vacuum-packed and heated in a water bath at $80^{\circ} \mathrm{C}$ to reach an inner temperature of $72^{\circ} \mathrm{C}$ and kept at the above temperature for a period of $90 \mathrm{~min}$. Following heat treatment, samples were cooled to room temperature and cuboids were excised measuring $10 \times 10 \times 40 \mathrm{~mm}$, which were subsequently cut perpendicular to muscle fibers. Additionally, sensory tenderness assessment according to a linear scale was carried out where a score of 10 points corresponded to very tender and 1 point very tough meat.

\section{Statistical analysis}

Gene expression at different stages was analyzed by one-way ANOVA followed by the Bonferroni test for pair-wise comparison. The co-expression genes were identified by Pearson correlation according to gene expression data during the development of longissimus dorsi muscle in cattle. The relationship of meat tenderness and gene expression was analyzed by the Pearson correlation at the significance level of 0.05 .

\section{RESULTS}

\section{Cloning and transcription factor binding site analysis in promoter regions of the ADAMTS4 and ADAMTS5 genes}

The 519-bp ADAMTS4 promoter region and 714-bp ADAMTS5 promoter region were cloned (Figure 1) and sequenced. Twelve transcription factor binding sites were found in the promoter region of the ADAMTS4 gene, including MZF1, SRY, Lyf-1, Ik-2, NF-kap and TATA. Six transcription factor binding sites were found in the promoter region of ADAMTS5 gene, including MZF1, C/EBPb, CdxA and Sox-5. 


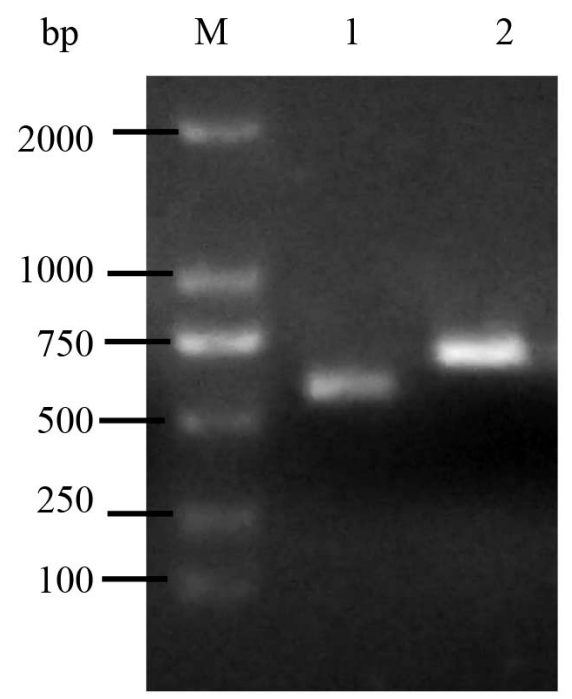

Figure 1. Agarose gel electrophoresis of ADAMTS4 and ADAMTS5 promoter regions. Lane $M=$ DL2000 DNA marker, lane 1 = ADAMTS4, lane 2 = ADAMTS5.

\section{Expression of ADAMTS4 and ADAMTS5 during muscle development}

The expression of ADAMTS4 was significantly higher in the longissimus dorsi muscle of 135-day and 185-day fetuses than in other developmental stages (Figure 2). The expression of ADAMTS5 showed a decreasing trend during the whole development, and the expression level in the 185-day fetus was significantly higher than in postnatal animals at $6,12,18$, 24 and 30 months (Figure 2).
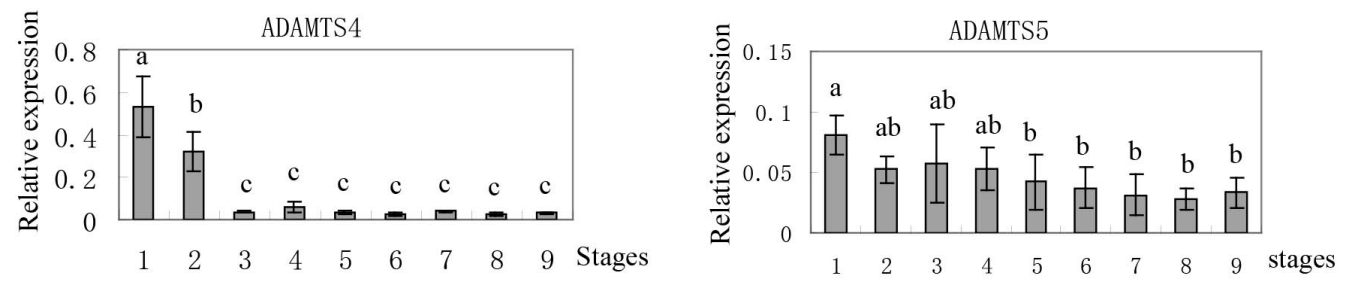

Figure 2. Expression of ADAMTS4 and ADAMTS5 during the development of longissimus dorsi muscle in Nanyang cattle. Treatments headed by the same letter denotes no significant difference $(\mathrm{P}>0.05)$. Column $1=$ pregnancy 135 day; column 2 = pregnancy 185 day; column 3 = pregnancy 280 day; column $4=3$ month old; column $5=6$ month old; column $6=12$ month old; column $7=18$ month old; column $8=24$ month old; column $9=30$ month old.

\section{Expression of MZF1, NF-kap and C/EBPb during muscle development}

Three muscle development-related transcriptional factors were selected to analyze their expression during the development of the longissimus dorsi muscle. The expression of MZF1 
was significantly higher in 135-day and 185-day fetuses than in other stages, which showed a similarity in expression pattern with ADAMTS4 (Figure3), and their correlation coefficient was $0.92(\mathrm{P}<0.01) . \mathrm{C} / \mathrm{EBPb}$ showed the opposite expression pattern as ADAMTS4 (Figure 3$)$ and their correlation coefficient was $-0.93(\mathrm{P}<0.01)$. The expression of NF-kap did not significantly change during the development of the longissimus dorsi muscle in Nanyang cattle (Figure 3).
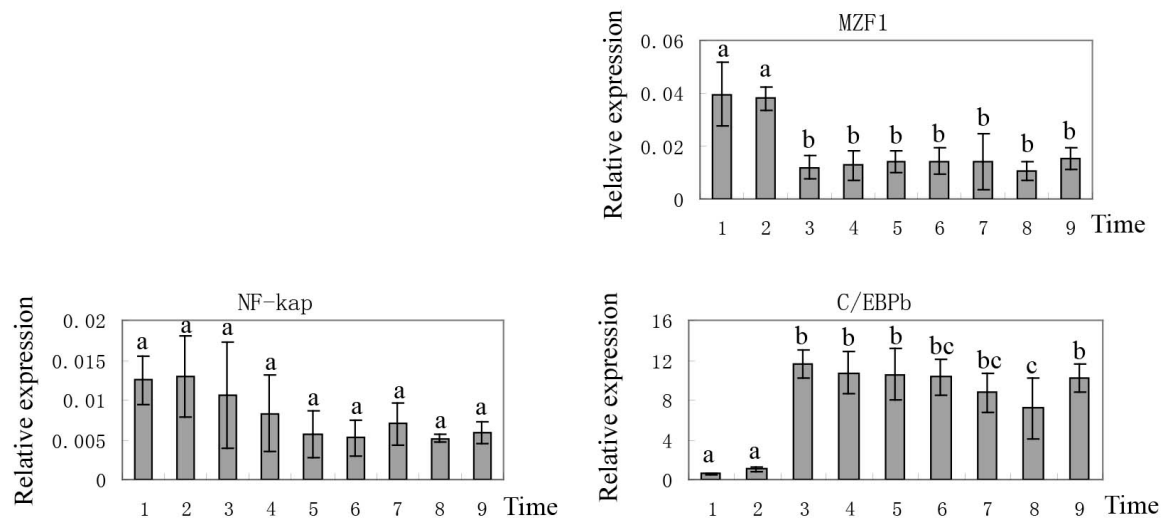

Figure 3. Expression of MZF1, NF-kap, and $\mathrm{C} / \mathrm{EBPb}$ during the development of longissimus dorsi muscle in Nanyang cattle. Treatments headed by the same letter denotes no significant difference $(\mathrm{P}>0.05)$. Column $1=$ pregnancy 135 day; column 2 = pregnancy 185 day; column $3=$ pregnancy 280 day; column $4=3$ month old; column $5=6$ month old; column $6=12$ month old; column $7=18$ month old; column $8=24$ month old; column $9=30$ month old.

\section{Relationships of ADAMTS4 and ADAMTS5 expression levels with meat tenderness in Nanyang cattle}

The mean tenderness of longissimus dorsi muscle samples of 24 Nanyang cattle was $4.94 \pm 1.12$, and the maximal tenderness and minimal tenderness were 7.10 and 3.11 , respectively. The Pearson correlation coefficient of ADAMTS4 expression levels with tenderness of longissimus dorsi muscle was $-0.3987(\mathrm{P}<0.05)$, which indicated that ADAMTS4 had a significant negative effective on the tenderness of longissimus dorsi muscle in cattle (Figure 4). The Pearson correlation coefficient of ADAMTS5 expression level with tenderness of longissimus dorsi muscle was $0.0193(\mathrm{P}>0.05)$, which indicated that the expression of ADAMTS5 had no significant relationship with the tenderness of longissimus dorsi muscle in cattle (Figure 4).
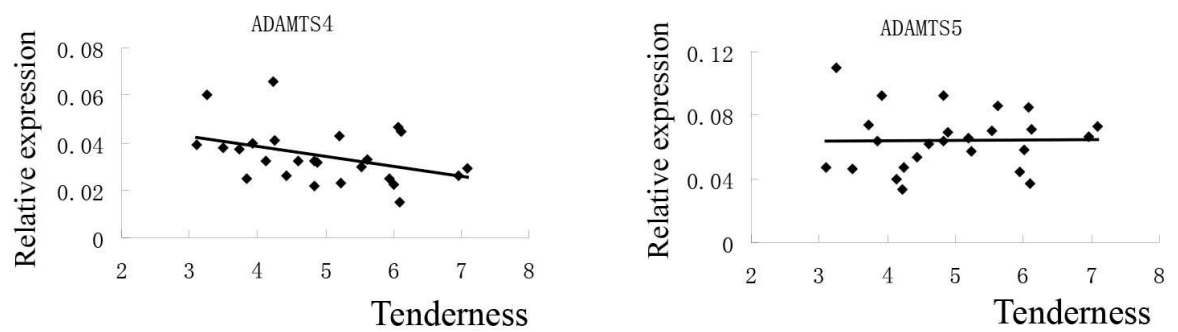

Figure 4. Correlation analysis of ADAMTS4 and ADAMTS5 expression with tenderness of longissimus dorsi muscle in Nanyang cattle. 


\section{DISCUSSION}

\section{Functions of ADAMTS4 and ADAMTS5 in the development of muscle tissue}

The ECM has an important effect on cell proliferation, migration and differentiation. Genes expression of ECM protein shows regulated changes during the development of muscle. The ADAMTS5 is expressed as early as E13.5 in skeletal muscle in mouse. Expression of ADAMTS5 during neuromuscular development and smooth muscle cells coincides with the broadly distributed proteoglycan versican, an ADAMTS5 substrate. The expression of versican showed a decreasing trend during the development of skeletal muscle in turkey (Sporer et al., 2011). In this study, the expression of ADAMTS5 also showed a decreasing trend with the development of longissimus dorsi muscle in Nanyang cattle. These observations suggest the major contexts in which developmental and physiological roles could be sought for this protease (McCulloch et al., 2009). Another major substrate of ADAMTS4 and ADAMTS5 protease is aggrecan, which is a kind of connective tissue macromolecule that provide a hydrated space around cells. The lack of aggrecan in the spontaneous mutant $\mathrm{cmd} / \mathrm{cmd}$ mouse strain leads to perinatal lethality owing to cleft palate and severe impairment of endochondral bone formation (Wai et al., 1998). Previous studies have demonstrated that the ECM comprising the connective tissue surrounding muscle can interact with growth factors, regulate cellular signal transduction pathways, and affect the growth and development of muscle fibers (Velleman, 2002).

Intramuscular fat (IMF) has an important effect on the sensory quality of culinary meat (Moeller et al., 2010). The level of IMF is dependent on breed, portion of meat in the carcass, slaughter weight and nutrition of the animal during the growing period. The adipose tissue belongs to connective tissue and the ADAMTS4 and ADAMTS5 have important effects on the proliferation and differentiation of preadipocytes by regulating the remodeling of ECM (Mariman and Wang, 2010). The expression of ADAMTS4 has shown a significant correlation with the IMF in Korean cattle (Lee et al., 2010), and this indicates the ADAMTS4 may affect meat tenderness by regulating the content of IMF, which is consistent with the results of our study.

\section{Relationship of transcription factor with gene expression regulation}

Transcription factors can regulate the expression of their targeted genes by accessing the cell nucleus and binding to TF binding sites, located in the promoter region of their targeted genes, activating or inhibiting its transcription. Importantly, such regulation acts in a species-, tissue- and time-specific manner, which turn on the right genes at the right time and in the right place (Levine and Tjian, 2003; Wasserman and Sandelin, 2004; Chen and Rajewsky, 2007). On the basis of this framework, many authors presumed that the genes regulated by the same transcription factors are more likely to show co-expression correlation and that, conversely, sets of genes showing a very high co-expression correlation are more likely to be regulated by the same set of transcription factors (Reverter and Chan, 2008). The relationship between gene co-expression and co-regulation has been explored in Drosophila melanogaster (Marco et al., 2009) and mouse (Kim et al., 2006). In this study, the expression pattern of MZF1 showed co-expression with the ADAMTS4, which indicated that the expression of ADAMTS4 may be regulated by MZF1 transcription factors. It was reported that MZF1 binding site was more 
likely to appear in the promoter region of muscle structural protein genes (Gu et al., 2011), such as FHL3 (a component of the muscle z-disc) (Takahashi et al., 2005). This study showed that the expression of ADAMTS4 may be regulated by MZF1 transcription factors.

The transcription factor binding sites of $\mathrm{MyoD}$, myogenin and other E-proteins were found in the exon 2 of ADAMTS5 gene and this indicated that the expression of ADAMTS5 may be regulated by myogenic regulatory factors (MRF). Exon 2 of the ADAMTS5 gene can also function as a very strong myogenic transcriptional enhancer in $\mathrm{C} 2 \mathrm{C} 12$ cells (Barthel and Liu, 2008). This indicated that gene expression is not only regulated by the promoter region, but also affected by exons in some specific genes.

\section{CONCLUSION}

The expression of ADAMTS4 was significantly higher in 135-day and 185-day fetuses than in other developmental stages, and the expression of ADAMTS5 showed a decreasing trend in Nanyang cattle longissimus dorsi muscle tissue from 135-day fetus to 30-monthold calf. The expression of ADAMTS4 may be positively regulated by MZF1 and negatively regulated by $\mathrm{C} / \mathrm{EBPb}$. The expression of ADAMTS4 showed a significant negative correlation with meat tenderness in Nanyang cattle.

\section{ACKNOWLEDGMENTS}

Research supported by China Agriculture (Cattle) Research System (CARS-38) and the major transgenics project during the 11th "Five Year Plan" (\#2011ZX08007-2).

\section{REFERENCES}

Apte SS (2004). A disintegrin-like and metalloprotease (reprolysin type) with thrombospondin type 1 motifs: the ADAMTS family. Int. J. Biochem. Cell Biol. 36: 981-985.

Barthel KK and Liu X (2008). A transcriptional enhancer from the coding region of ADAMTS5. PLoS One 3: e2184.

Cánovas A, Quintanilla R, Amills M and Pena RN (2010). Muscle transcriptomic profiles in pigs with divergent phenotypes for fatness traits. BMC Genomics 11: 372 .

Chen K and Rajewsky N (2007). The evolution of gene regulation by transcription factors and microRNAs. Nat. Rev. Genet. 8: 93-103.

Durigova M, Troeberg L, Nagase H, Roughley PJ, et al. (2011). Involvement of ADAMTS5 and hyaluronidase in aggrecan degradation and release from OSM-stimulated cartilage. Eur. Cell Mater. 21: 31-45.

Fosang AJ and Rogerson FM (2010). Identifying the human aggrecanase. Osteoarthritis Cartilage 18: 1109-1116.

Gillies AR and Lieber RL (2011). Structure and function of the skeletal muscle extracellular matrix. Muscle Nerve 44: 318-331.

Gu Q, Nagaraj SH, Hudson NJ, Dalrymple BP, et al. (2011). Genome-wide patterns of promoter sharing and co-expression in bovine skeletal muscle. BMC Genomics 12: 23.

Hojilla CV, Wood GA and Khokha R (2008). Inflammation and breast cancer: metalloproteinases as common effectors of inflammation and extracellular matrix breakdown in breast cancer. Breast Canc. Res. 10: 205.

Iwanowska A, Grzes B, Mikolajczak B, Iwanska E, et al. (2011). Impact of polymorphism of the regulatory subunit of the mu-calpain (CAPN1S) on the proteolysis process and meat tenderness of young cattle. Mol. Biol. Rep. 38: $1295-1300$.

Kim RS, Ji H and Wong WH (2006). An improved distance measure between the expression profiles linking co-expression and co-regulation in mouse. BMC Bioinformatics 7: 44.

Lee SH, Gondro C, van der Werf J, Kim NK, et al. (2010). Use of a bovine genome array to identify new biological pathways for beef marbling in Hanwoo (Korean Cattle). BMC Genomics 11: 623.

Levine M and Tjian R (2003). Transcription regulation and animal diversity. Nature 424: 147-151. 
Livak KJ and Schmittgen TD (2001). Analysis of relative gene expression data using real-time quantitative PCR and the $2{ }^{-\Delta \Delta C}(\mathrm{~T})$ method. Methods 25: 402-408.

Marco A, Konikoff C, Karr TL and Kumar S (2009). Relationship between gene co-expression and sharing of transcription factor binding sites in Drosophila melanogaster. Bioinformatics 25: 2473-2477.

Mariman EC and Wang P (2010). Adipocyte extracellular matrix composition, dynamics and role in obesity. Cell Mol. Life Sci. 67: 1277-1292.

Matthews RT, Gary SC, Zerillo C, Pratta M, et al. (2000). Brain-enriched hyaluronan binding (BEHAB)/brevican cleavage in a glioma cell line is mediated by a disintegrin and metalloproteinase with thrombospondin motifs (ADAMTS) family member. J. Biol. Chem. 275: 22695-22703.

McCulloch DR, Le Goff C, Bhatt S, Dixon LJ, et al. (2009). Adamts5, the gene encoding a proteoglycan-degrading metalloprotease, is expressed by specific cell lineages during mouse embryonic development and in adult tissues. Gene Expr. Patterns 9: 314-323.

Moeller SJ, Miller RK, Aldredge TL, Logan KE, et al. (2010). Trained sensory perception of pork eating quality as affected by fresh and cooked pork quality attributes and end-point cooked temperature. Meat Sci. 85: 96-103.

Porter S, Clark IM, Kevorkian L and Edwards DR (2005). The ADAMTS metalloproteinases. Biochem. J. 386: 15-27.

Reverter A and Chan EK (2008). Combining partial correlation and an information theory approach to the reversed engineering of gene co-expression networks. Bioinformatics 24: 2491-2497.

Roughley PJ (2001). Articular cartilage and changes in arthritis: noncollagenous proteins and proteoglycans in the extracellular matrix of cartilage. Arthritis Res. 3: 342-347.

Sandy JD, Westling J, Kenagy RD, Iruela-Arispe ML, et al. (2001). Versican V1 proteolysis in human aorta in vivo occurs at the Glu441-Ala442 bond, a site that is cleaved by recombinant ADAMTS-1 and ADAMTS-4. J. Biol. Chem. 276: 13372-13378.

Seals DF and Courtneidge SA (2003). The ADAMs family of metalloproteases: multidomain proteins with multiple functions. Genes Dev. 17: 7-30.

Sporer KR, Tempelman RJ, Ernst CW, Reed KM, et al. (2011). Transcriptional profiling identifies differentially expressed genes in developing turkey skeletal muscle. BMC Genomics 12: 143.

Takahashi K, Matsumoto C and Ra C (2005). FHL3 negatively regulates human high-affinity IgE receptor $\beta$-chain gene expression by acting as a transcriptional co-repressor of MZF-1. Biochem. J. 386: 191-200.

Velleman SG (2002). Role of the extracellular matrix in muscle growth and development. J. Anim. Sci. 80: E8-E13.

Wai AW, Ng LJ, Watanabe H, Yamada Y, et al. (1998). Disrupted expression of matrix genes in the growth plate of the mouse cartilage matrix deficiency (cmd) mutant. Dev. Genet. 22: 349-358.

Wasserman WW and Sandelin A (2004). Applied bioinformatics for the identification of regulatory elements. Nat. Rev. Genet. 5: 276-287.

Yanagishita M (1993). Function of proteoglycans in the extracellular matrix. Acta Pathol. Jpn. 43: 283-293.

Yang MQ and Elnitski LL (2008). Diversity of core promoter elements comprising human bidirectional promoters. BMC Genomics 9 (Suppl 2): S3. 